\section{OVERCOMING RESISTANCE TO ANTI-PD-1 WITH TUMOR AGNOSTIC NBTXR3: FROM BENCH TO BED SIDE}

${ }^{1}$ James Welsh*, ${ }^{2}$ Colette Shen, ${ }^{3}$ Jessica Frakes, ${ }^{4}$ Jiaxin Niu, ${ }^{2}$ Jared Weiss, ${ }^{3}$ Jimmy Caudell, ${ }^{5} \mathrm{Hu}$ Yun, ${ }^{5} \mathrm{Hampartsoum} \mathrm{Barsoumian,}{ }^{6}$ Juliette Thariat, ${ }^{7}$ Sylvie Bonvalot, ${ }^{8}$ Zsusanna Papaï, ${ }^{5}$ Maria Angelica Cortez, ${ }^{9}$ Ping Zhang, ${ }^{10}$ Katherine Jameson, ${ }^{9}$ Patricia Said, ${ }^{9}$ Sébastien Paris, ${ }^{11}$ Tanguy Seiwert. 'University of Texas MD Anderson Cancer, Houston, TX, USA; ${ }^{2}$ University of North Carolina, Chapel Hill, NC, USA; ${ }^{3}$ Moffitt Cancer Center, Tampa, USA; ${ }^{4}$ Banner MD Anderson Cancer Center, Gilbert, AZ, USA; ${ }^{5}$ MD Anderson Cancer Center, Houston, TX, USA; ${ }^{6}$ Centre Baclesse, Caen, Cedex 2, France; ${ }^{7}$ Institut Curie, Paris, Cedex 05, France; ${ }^{8} \mathrm{MH}$ Egeszsegugyi Kozpont, Hungarian Defe, Budapest, Hungary; ${ }^{9}$ Nanobiotix, SA, Paris, France; ${ }^{10}$ Nanobiotix, corp, Cambridge, MA, USA; ${ }^{11}$ John Hopkins Medecine, Baltimore, Maryland, USA

Background Despite recent advances, resistance to immune checkpoint inhibitors (ICI), observed in over $80 \%$ of treated patients, is currently the main challenge immuno-oncology is facing. Intense efforts are being made to identify combination therapies that could improve ICI response rates. Administered intratumorally, NBTXR3 enhances the energy dose deposited by ionizing radiation within tumor cells, increasing the antitumor efficacy of radiation therapy (XRT) without adding toxicity to surrounding tissues. Here we present evidence that NBTXR3 activated by XRT primes the immune system, producing an anti-tumor response, including activation of the cGAS-STING pathway, that overcomes anti-PD-1 resistance both in mice models and patients.

Methods Abscopal assays were conducted in immunocompetent mice. Tumor cell lines, sensitive or resistant to anti-PD-1, were injected in both flanks of mice. Intratumoral injection of NBTXR3 (or vehicle) followed by XRT was performed in right flank (primary) tumors only. Some mice also received anti-PD-1 injections. Tumor growth was monitored, and tumor immune cell infiltrates were analyzed by immunohistochemistry (IHC). Separately, in the phase II/III randomized trial Act.in. Sarc [NCT02379845] patients with locally advanced soft tissue sarcoma (STS) received either NBTXR3+XRT or XRT alone followed by wide tumor resection. Pre- and post-treatment tumor samples from patients in both groups were analyzed by IHC and Digital Pathology for immune biomarkers. The safety and efficacy (RECIST 1.1/iRECIST) of NBTXR3 plus stereotactic ablative radiotherapy (SABR) in combination with antiPD-1 is being evaluated in three cohorts of patients with advanced cancers [NCT03589339].

Results Pre-clinical studies demonstrated that NBTXR3+XRT induces an immune response a not observed with XRT alone and enhances systemic control. IHC showed significant increase of CD8 + T-cell infiltrates in both NBTXR3 +XRT treated and untreated tumors compared to XRT alone. Similarly, increased CD8 + T-cell density (pre- vs post-treatment) was observed in tumor tissues from STS patients treated with NBTXR3+XRT. Tumor samples from the NBTXR3+XRT group also displayed increased PD-1+ cell density. Furthermore, in combination with anti-PD-1, NBTXR3+XRT improved local and systemic control in mice bearing anti-PD-1 resistant lung tumors, as well as resulted in reduced number of spontaneous lung metastases.Preliminary efficacy data from the first in human trial of NBTXR3+XRT in combination with anti-PD-1 showed tumor response in patients who progressed on prior anti-PD-1.

Conclusions The clinical efficacy of NBTXR3+XRT has been demonstrated as a single agent. We now demonstrate that it potentiates anti-PD-1 treatment to overcome resistance mechanisms. These results highlight the potential of NBTXR3 + XRT to positively impact the immuno-oncology field.
Ethics Approval This study was approved by local institution's review board

http://dx.doi.org/10.1136/jitc-2020-SITC2020.0396

\section{INTRA-TUMOR IMMUNOTHERAPY INJECTIONS UTILIZING IMAGE GUIDANCE IN INTERVENTIONAL RADIOLOGY: CLINICAL TRIAL EXPERIENCE AT A TERTIARY CARE CANCER CENTER}

Ravi Murthy*, Rahul Sheth, Alda Tam, Sanjay Gupta, Vivek Subbiah, Filip Janku, Timothy Yap, Adi Diab, Aung Naing, Sapna Patel, Funda Meric-Bernstam. MD Anderson Cancer Center, Houston, TX, USA

Background Image guided intra-tumor administration of investigational immunotherapeutic agents represents an expanding field of interest. We present a retrospective review of the safety, feasibility \& technical nuances of real-time image guidance for injection \& biopsy across a spectrum of extracranial solid malignancies utilizing the discipline of Interventional Radiology.

Methods Patients who were enrolled in image guided intratumoral immunotherapy injection (ITITI) clinical trials over a 6 year period (2013-19) at a single tertiary care cancer center were included in this analysis. Malignancy, location, imaging guidance utilized for ITITI \& biopsy for injected (adscopal) \& non-injected (abscopal) lesions were determined and categorized. Peri-procedural adverse events were noted.

Results 262 pts (146 female, 61 yrs median) participating in 29 immunotherapeutic clinical trials (TLR \& STING agonists, gene therapy, anti CD-40, viral/bacterial/metabolic oncolytics) met study criteria. Malignancies included melanoma 88, sarcoma 32, colorectal 29, breast 23, lung 17 , head $\&$ neck 15 , ovarian 8 , neuroendocrine 7 , pancreatic adenocarcinoma 6,3 each (cholangioCA, endometrial, bladder, GI tract), 2 each (RCC, thymicCA, lymphoma, merkel cell, prostate) \& others 1 each (CUP, GIST, dermatofibrosarcoma, DSRT, neuroblastoma, thyroid). All $169 \& 93$ patients received the intended 1371 ITITI in parietal (abdominal/chest wall, extremity, neck, pelvis) or visceral (liver, lung, peritoneum, adrenal) locations respectively; 83 patients received lymph node injections within either location. Imaging guidance was US in $68 \%$ of the cohort (US 161, CT+US 19); CT was used in 30\% (81) \& MRI in 1 patient. Median diameter of the ITITI lesion was $32 \mathrm{~mm}(8-230 \mathrm{~mm})$. Median volume of the ITITI therapeutic material/session was $2 \mathrm{ml}(1-6.9 \mathrm{ml})$. Lesions were accessed using a coaxial technique. ITITI delivery needles used at operator preference \& tailored to lesion characteristics were either a 21G/22G Chiba, 21G Profusion (Cook Medical), 22G Morrison (AprioMed), 25G hypodermic (BD) \& 18G Quadrafuse (Rex Medical). 2840 core biopsies (>18G Tru-cut core, Mission, Bard Medical) were performed in 237 patients during 690 procedures; biopsy sessions were often concurrent \& of the ITITI site. 137 patients also underwent biopsy of a nonITITI site (89 parietal location). Dimensions of the non-ITITI lesion were median $10 \mathrm{~mm}(7-113 \mathrm{~mm})$; US image guidance was used in 97 patients (72\%) to obtain a total of 1257 , $>18 \mathrm{G}$ Tru-core samples. $1.3 \%$ of injections resulted in SAE (NCI CTC AE >3) and $0.5 \%$ of 4097 biopsies developed major complications (SIR Criteria); both categories were manageable.

Conclusions Utilizing real time image guidance, ITITI to the administration of a myriad of investigational 